\title{
Existence of Positive Periodic Solutions of a Lotka-Volterra System with Multiple Time Delays
}

\author{
Changjin $\mathrm{Xu}$ \\ Faculty of Science, Hunan Institute of Engineering \\ Xiangtan 411004, China \\ Department of Mathematics, Central South University \\ Changsha 410083, China \\ Tel: 86-732-268-0745 E-mail: xcj403@126.com \\ Maoxin Liao \\ Department of Mathematics, Central South University \\ Changsha 410083, China \\ School of Mathematics and Physics, Nanhua University \\ Hengyang 421001, China
}

The research is supported by National Natural Science Foundation of China (No. 10771215)

\begin{abstract}
In this paper, a class of Lotka-Volterra system with multiple time delays is considered. By using the continuation theorem of coincidence degree theory, we derive a set of easily verifiable sufficient conditions that guarantees the existence of at least a positive periodic solution.
\end{abstract}

Keywords: Lotka-Volterra system, Periodic solution, Multiple time delay, Continuation theorem, Topological degree

\section{Introduction}

Lotka-Volterra system is an important population system and has been studied by many authors, see (Chen Shihua, et.al, 2004, Fan Meng, et.al,1999, Li Yongkun, et.al, 2001) and the reference therein. but most of the previous results focused on the stability, attractiveness, persistence and periodicity of solution to the ordinary differential systems or time delay systems with constant delays. Rare work has been done for the systems with varying delays and varying coefficients. In 1991, Weng di Wang et. al (Wang Wending,et. al, 1991) had considered a two-dimensional predator-prey system with a finite constant number discrete delays

$$
\left\{\begin{array}{l}
\dot{x}(t)=x(t)\left[r_{1}-\sum_{j=1}^{m} a_{1 j} x\left(t-\tau_{1 j}\right)-\sum_{j=1}^{m} b_{1 j} y\left(t-\rho_{1 j}\right)\right], \\
\dot{y}(t)=y(t)\left[r_{2}+\sum_{j=1}^{m} a_{2 j} x\left(t-\tau_{2 j}\right)-\sum_{j=1}^{m} b_{2 j} y\left(t-\rho_{2 j}\right)\right],
\end{array}\right.
$$

with initial conditions

$$
\begin{aligned}
& x(s)=\varphi(s) \geq 0, s \in[-\tau, 0] ; \varphi(0)>0, \\
& y(s)=\psi(s) \geq 0, s \in[-\tau, 0] ; \psi(0)>0,
\end{aligned}
$$

where $r_{1}, r_{2}$ are real constants with $r_{1}>0 ; a_{i j}, b_{i j}, \tau_{i j}, \rho_{i j}(i=1,2 ; j=1,2, \ldots, m)$ are non-negative constants. Not all of $a_{1 j}$ and not all $b_{1 j}(j=1,2, \ldots, m)$ are zero; Both $\varphi(s)$ and $\psi(s)$ are continuous on the interval $[-\tau, 0]$ in which $\tau=\max \left\{\tau_{i j}, \rho_{i j}: i=1,2 ; j=1,2, \ldots, m\right\}$. And obtained the conclusion that the time delays are harmless for uniform persistence of the solutions to the system. 
We note that any biological or environmental parameters are naturally subject to fluctuation in time. It is necessary and important to consider models with periodic ecological parameters or perturbations which might be naturally exposed ( for example, those due to seasonal effects of weather, food supply, mating habits, hunting or harvesting seasons, etc.). Thus, the assumption of periodicity of the parameters is a way of incorporating the periodicity of the environment.

In this paper, we are concerned with the effects of periodicity of ecological and environmental parameters and time delays. Then system (1) can be modified as the form:

$$
\left\{\begin{array}{l}
\dot{x}(t)=x(t)\left[r_{1}(t)-\sum_{j=1}^{m} a_{1 j}(t) x\left(t-\tau_{1 j}(t)\right)-\sum_{j=1}^{m} b_{1 j}(t) y\left(t-\rho_{1 j}(t)\right)\right], \\
\dot{y}(t)=y(t)\left[r_{2}(t)+\sum_{j=1}^{m} a_{2 j}(t) x\left(t-\tau_{2 j}(t)\right)-\sum_{j=1}^{m} b_{2 j}(t) y\left(t-\rho_{2 j}(t)\right)\right]
\end{array}\right.
$$

with initial conditions

$$
\left\{\begin{array}{l}
x(s)=\varphi(s) \geq 0, s \in[-\tau, 0] ; \varphi(0)>0, \\
y(s)=\psi(s) \geq 0, s \in[-\tau, 0] ; \psi(0)>0,
\end{array}\right.
$$

where $r_{1}(t), r_{2}(t)$ are real functions with $r_{i}(t)>0,(i=1,2) ; a_{i j}(t), b_{i j}(t), \tau_{i j}(t), \rho_{i j}(t)(i=1,2 ; j=1,2, \ldots, m)$ are nonnegative functions. Not all of $a_{1 j}(t)$ and not all $b_{1 j}(t)(j=1,2, \ldots, m)$ are zero; Both $\varphi(s)$ and $\psi(s)$ are continuous on the interval $[-\tau, 0]$ in which $\tau=\max _{t \in R} \max \left\{\tau_{i j}(t), \rho_{i j}(t): i=1,2 ; j=1,2, \ldots, m\right\}$.

Throughout the paper, we always assume that

$\left(H_{1}\right) r_{i}(t), a_{i j}(t), b_{i j}(t), \tau_{i j}(t), \rho_{i j}(t)(i=1,2 ; j=1,2, \ldots, m)$ are $\omega$ periodic, i.e.,

$$
\begin{gathered}
r_{i}(t+\omega)=r_{i}(t), a_{i j}(t+\omega)=a_{i j}(t), b_{i j}(t+\omega)=b_{i j}(t), \\
\tau_{i j}(t+\omega)=\tau_{i j}(t), \rho_{i j}(t+\omega)=\rho_{i j}(t)
\end{gathered}
$$

for any $t \in R$.

$\left(H_{2}\right) r_{i}(t), a_{i j}(t), b_{i j}(t), \tau_{i j}(t), \rho_{i j}(t)(i=1,2 ; j=1,2, \ldots, m)$ are all positive, i.e.

$$
r_{i}(t), a_{i j}(t), b_{i j}(t), \tau_{i j}(t), \rho_{i j}(t)(i=1,2 ; j=1,2, \ldots, m)>0 .
$$

The principle object of this article is to find a set of sufficient conditions that guarantees the existence of at least a positive periodic solution for system (2) (3).

\section{Basic lemma}

In order to explore the existence of positive periodic solutions of (2) (3) and for the reader's convenience, we shall first summarize below a few concepts and results without proof, borrowing from (Yang Zhihui, et. al, 2007).

Let $X, Y$ be normed vector spaces, $L: D o m L \subset X \rightarrow Y$ is a linear mapping, $N: X \rightarrow Y$ is a continuous mapping. The mapping $L$ will be called a Fredholm mapping of index zero if $\operatorname{dim} \operatorname{Ker} L=\operatorname{codim} \operatorname{Im} L<+\infty$ and $\operatorname{ImL}$ is closed in $Y$. If $L$ is a Fredholm mapping of index zero and there exist continuous projectors $P: X \rightarrow X$ and $Q: Y \rightarrow Y$ such that $\operatorname{Im} P=\operatorname{Ker} L, \operatorname{Im} L=\operatorname{Ker} Q=\operatorname{Im}(I-Q)$, it follows that $L \mid \operatorname{Dom} L \cap \operatorname{Ker} P:(I-P) X \rightarrow \operatorname{Im} L$ is invertible. We denote the inverse of that map by $K_{P}$. If $\Omega$ is an open bounded subset of $X$, the mapping $N$ will be called $L-$ compact on $\bar{\Omega}$ if $Q N(\bar{\Omega})$ is bounded and $K_{P}(I-Q) N: \bar{\Omega} \rightarrow X$ is compact. Since $\operatorname{Im} Q$ is isomorphic to $K e r L$, there exist isomorphisms $J: \operatorname{Im} Q \rightarrow \operatorname{KerL}$.

Lemma 2.1. (Robert E. Gaines et. al, 1991)(Continuation Theorem ) Let L be a fredholm mapping of index zero and let $N$ be L-compact on $\bar{\Omega}$. Suppose

(a) for each $\lambda \in(0,1)$, every solution $x$ of $L x=\lambda N x$ is such that $x \notin \partial \Omega$;

(b) $Q N x \neq 0$ for each $x \in \operatorname{Ker} L \cap \partial \Omega$, and $\operatorname{deg}\{J Q N, \Omega \cap \partial \operatorname{Ker} L, 0\} \neq 0$;

Then the equation $L x=N x$ has at least one solution lying in DomL $\cap \bar{\Omega}$.

Lemma 2.2. $R_{+}^{2}=\left\{\left((x(t), y(t))^{T} \in R^{2} \mid x(t)>0, y(t)>0\right\}\right.$ is positive invariant with respect to system (2) (3).

Proof. In fact,

$$
\begin{aligned}
& x(t)=\varphi(0) \exp \int_{0}^{t}\left[r_{1}(s)-\sum_{j=1}^{m} a_{1 j}(s) x\left(s-\tau_{1 j}(s)\right)-\sum_{j=1}^{m} b_{1 j}(s) y\left(s-\rho_{1 j}(s)\right)\right] d s, \\
& y(t)=\psi(0) \exp \int_{0}^{t}\left[r_{2}(s)+\sum_{j=1}^{m} a_{2 j}(s) x\left(s-\tau_{2 j}(s)\right)-\sum_{j=1}^{m} b_{2 j}(s) y\left(s-\rho_{2 j}(s)\right)\right] d s .
\end{aligned}
$$

In view of $\varphi(0)>0, \psi(0)>0,(i=1,2)$, obviously, the conclusion follows. 


\section{Existence of positive periodic solutions}

For convenience and simplicity in the following discussion, we always use the notations below throughout the paper:

$$
\bar{g}=\frac{1}{\omega} \int_{0}^{\omega} g(t) d t, g^{L}=\min _{t \in[0, \omega]} g(t), g^{M}=\max _{t \in[0, \omega]} g(t),
$$

where $g(t)$ is a $\omega$ continuous periodic function. Let $\sigma_{i j}(t)=t-\tau_{i j}(t), \theta_{i j}(t)=t-\rho_{i j}(t), t \in R, i=1,2 ; j=1,2, \ldots, m$. Assume that

$$
\left(H_{3}\right) \tau_{i j}^{\prime}(t)<1, \rho_{i j}^{\prime}(t)<1,(i=1,2 ; j=1,2, \ldots, m)
$$

Then $\sigma_{i j}(t)$ and $\theta_{i j}(t)$ have inverse functions denoted by $\mu_{i j}(t), \varepsilon_{i j}(t),(i=1,2 ; j=1,2, \ldots, m)$, respectively. Obviously, $\mu_{i j}(t+\omega)=\mu_{i j}(t)+\omega, \varepsilon_{i j}(t+\omega)=\varepsilon_{i j}(t)+\omega$.

In the following, we will ready to state and prove our result.

Theorem 3.1. Suppose that $\left(H_{1}\right),\left(H_{2}\right),\left(H_{3}\right),\left(H_{4}\right) D_{22}^{L}{\overline{r_{1}}}^{L}>D_{12}^{M}{\overline{r_{2}}}^{M}$ and

$\left(H_{5}\right) \overline{r_{1}} \sum_{j=1}^{m} \overline{b_{2 j}}>\overline{r_{2}} \sum_{j=1}^{m} \overline{b_{1 j}}$ hold, where

$$
D_{22}=\sum_{j=1}^{m} \frac{b_{2 j}(t) \varepsilon_{2 j}(t)}{1-\rho_{2 j}^{\prime}\left(\varepsilon_{2 j}(t)\right)}, D_{12}=\sum_{j=1}^{m} \frac{b_{1 j}(t) \varepsilon_{1 j}(t)}{1-\rho_{1 j}^{\prime}\left(\varepsilon_{1 j}(t)\right)},
$$

then the system (2) (3) has at least a w periodic solution.

Proof. Since solutions of (2) (3) remained positive for all $t \geq 0$, we let

$$
u_{1}(t)=\ln [x(t)], u_{2}(t)=\ln [y(t)] .
$$

Substituting (4) into (2), we obtain

$$
\left\{\begin{array}{l}
\dot{u_{1}}(t)=r_{1}(t)-\sum_{j=1}^{m} a_{1 j}(t) \exp \left\{u_{1}\left(t-\tau_{1 j}(t)\right)\right\}-\sum_{j=1}^{m} b_{1 j}(t) \exp \left\{u_{2}\left(t-\rho_{1 j}(t)\right)\right\} \\
\dot{u_{2}}(t)=r_{2}(t)+\sum_{j=1}^{m} a_{2 j}(t) \exp \left\{u_{1}\left(t-\tau_{2 j}(t)\right)\right\}-\sum_{j=1}^{m} b_{2 j}(t) \exp \left\{u_{2}\left(t-\rho_{2 j}(t)\right)\right\}
\end{array}\right.
$$

It is easy to see that if system (5) has one $\omega$ periodic solution $\left(u_{1}^{*}(t), u_{2}^{*}(t)\right)^{T}$, then $\left(x^{*}(t), y *(t)\right)^{T}=\left(\exp \left[u_{1}^{*}(t), \exp \left[u_{2}^{*}(t)\right]\right)^{T}\right.$ is a positive solution of system (2). Therefore, to complete the proof, it suffices to show that system (5) has at least one $\omega$ periodic solution.

Let $X=Z=\{u(t)\}=\left\{\left(u_{1}(t), u_{2}(t)\right)^{T} \mid u(t) \in C\left(R, R^{2}\right), u(t+\omega)=u(t)\right\}$, and define $\|u\|=\left\|\left(u_{1}(t), u_{2}(t)\right)^{T}\right\|=\max _{t \in[0, \omega]}\left|u_{1}(t)\right|+$ $\max _{t \in[0, \omega]}\left|u_{2}(t)\right|$. Then $X$ and $Z$ are Banach spaces when they are endowed with the norm $\|$.$\| .$

Let $L: D o m L \subset X \rightarrow Z$ and $N: X \rightarrow Z$ be the following:

$$
N u=\left(\begin{array}{c}
L u=x^{\prime}(t), \\
r_{1}(t)-\sum_{j=1}^{m} a_{1 j}(t) \exp \left\{u_{1}\left(t-\tau_{1 j}(t)\right)\right\}-\sum_{j=1}^{m} b_{1 j}(t) \exp \left\{u_{2}\left(t-\rho_{1 j}(t)\right)\right\} \\
r_{2}(t)+\sum_{j=1}^{m} a_{2 j}(t) \exp \left\{u_{1}\left(t-\tau_{2 j}(t)\right)\right\}-\sum_{j=1}^{m} b_{2 j}(t) \exp \left\{u_{2}\left(t-\rho_{2 j}(t)\right)\right\}
\end{array}\right) .
$$

Define continuous projective operators $P$ and $Q$ :

$$
P u=\frac{1}{\omega} \int_{0}^{\omega} u(t) d t, Q u=\frac{1}{\omega} \int_{0}^{\omega} u(t) d t, u \in X, u \in Z .
$$

We can see that $\operatorname{Ker} L=\left\{u \in X \mid u=h \in R^{2}\right\}, \operatorname{Im} L=\left\{u \in Z \mid \int_{0}^{\omega} u(t) d t=0\right\}$ is closed in $X$ and $\operatorname{dim}(\operatorname{KerL})=2=$ $\operatorname{codim}(\operatorname{Im} L)$, then it follows that $L$ is a fredholm mapping of index zero. Moreover, it is easy to check that

$$
Q N x=\left(\begin{array}{c}
\frac{1}{\omega} \int_{0}^{\omega}\left[r_{1}(t)-\sum_{j=1}^{m} a_{1 j}(t) \exp \left\{u_{1}\left(t-\tau_{1 j}(t)\right)\right\}-\sum_{j=1}^{m} b_{1 j}(t) \exp \left\{u_{2}\left(t-\rho_{1 j}(t)\right)\right\}\right] d t \\
\frac{1}{\omega} \int_{0}^{\omega}\left[r_{2}(t)+\sum_{j=1}^{m} a_{2 j}(t) \exp \left\{u_{1}\left(t-\tau_{2 j}(t)\right)\right\}-\sum_{j=1}^{m} b_{2 j}(t) \exp \left\{u_{2}\left(t-\rho_{2 j}(t)\right)\right\}\right] d t
\end{array}\right) .
$$


By easily computation, we have

$$
K_{P}(z)=\int_{0}^{\omega} z(u) d u-\frac{1}{\omega} \int_{0}^{\omega}\left[\int_{0}^{t} z(u) d u\right] d t
$$

$K_{P}(I-Q) N u=$

$$
\left(\begin{array}{c}
\int_{0}^{t} F_{1}(s) d s \\
\int_{0}^{t} F_{2}(s) d s
\end{array}\right)-\left(\begin{array}{c}
\frac{1}{\omega} \int_{0}^{\omega} \int_{0}^{t} F_{1}(s) d s d t \\
\frac{1}{\omega} \int_{0}^{\omega} \int_{0}^{t} F_{2}(s) d s d t
\end{array}\right)-\left(\begin{array}{c}
\left(\frac{t}{\omega}-\frac{1}{2}\right) \int_{0}^{\omega} F_{1}(s) d s \\
\left(\frac{t}{\omega}-\frac{1}{2}\right) \int_{0}^{\omega} F_{2}(s) d s
\end{array}\right)
$$

where

$$
\begin{aligned}
& F_{1}(s)=r_{1}(s)-\sum_{j=1}^{m} a_{1 j}(s) \exp \left\{u_{1}\left(s-\tau_{1 j}(s)\right)\right\}-\sum_{j=1}^{m} b_{1 j}(s) \exp \left\{u_{2}\left(s-\rho_{1 j}(s)\right)\right\}, \\
& F_{2}(s)=r_{2}(s)+\sum_{j=1}^{m} a_{2 j}(s) \exp \left\{u_{1}\left(s-\tau_{2 j}(s)\right)\right\}-\sum_{j=1}^{m} b_{2 j}(s) \exp \left\{u_{2}\left(s-\rho_{2 j}(s)\right)\right\} .
\end{aligned}
$$

Obviously, $Q N$ and $K_{P}(I-Q) N$ are continuous. Since $X$ is a finite-dimensional Banach space, using the Ascoli-Arzela theorem, it is not difficult to show that $\overline{K_{P}(I-Q) N(\bar{\Omega})}$ is compact for any open bounded set $\Omega \subset X$. Moreover, $Q N(\bar{\Omega})$ is bounded. Thus, $N$ is $L$-compact on $\bar{\Omega}$ with any open bounded set $\Omega \subset X$.

Now we are at the point to search for an appropriate open, bounded subset $\Omega$ for the application of the continuation theorem. Corresponding to the operator equation $L y=\lambda N y, \lambda \in(0,1)$, we have

$$
\left\{\begin{array}{l}
\dot{u_{1}}(t)=\lambda\left[r_{1}(t)-\sum_{j=1}^{m} a_{1 j}(t) \exp \left\{u_{1}\left(t-\tau_{1 j}(t)\right)\right\}-\sum_{j=1}^{m} b_{1 j}(t) \exp \left\{u_{2}\left(t-\rho_{1 j}(t)\right)\right\}\right], \\
\dot{u_{2}}(t)=\lambda\left[r_{2}(t)+\sum_{j=1}^{m} a_{2 j}(t) \exp \left\{u_{1}\left(t-\tau_{2 j}(t)\right)\right\}-\sum_{j=1}^{m} b_{2 j}(t) \exp \left\{u_{2}\left(t-\rho_{2 j}(t)\right)\right\}\right] .
\end{array}\right.
$$

Suppose that $u(t)=\left(u_{1}(t), u_{2}(t)\right)^{T} \in X$ is an arbitrary solution of system (8) for a certain $\lambda \in(0,1)$, integrating both sides of $(8)$ over the interval $[0, \omega]$ with respect to $t$, we obtain

$$
\left\{\begin{array}{l}
\int_{0}^{t}\left[\sum_{j=1}^{m} a_{1 j}(t) \exp \left\{u_{1}\left(t-\tau_{1 j}(t)\right)\right\}+\sum_{j=1}^{m} b_{1 j}(t) \exp \left\{u_{2}\left(t-\rho_{1 j}(t)\right)\right\}\right] d t=\overline{r_{1}} \omega \\
\int_{0}^{t}\left[\sum_{j=1}^{m} a_{2 j}(t) \exp \left\{u_{1}\left(t-\tau_{2 j}(t)\right)\right\}-\sum_{j=1}^{m} b_{2 j}(t) \exp \left\{u_{2}\left(t-\rho_{2 j}(t)\right)\right\}\right] d t=-\overline{r_{2}} \omega
\end{array}\right.
$$

In view of the following:

$$
\begin{aligned}
\int_{0}^{\omega} & \sum_{j=1}^{m} a_{i j}(t) \exp \left\{u_{1}\left(t-\tau_{i j}(t)\right)\right\} d t \\
= & \int_{-\tau_{i j}(0)}^{\omega-\tau_{i j}(\omega)} \sum_{j=1}^{m} \frac{a_{i j}\left(\mu_{i j}(s)\right)}{1-\tau_{i j}^{\prime}\left(\mu_{i j}(s)\right)} \exp \left\{u_{1}(s)\right\} d s \\
= & \int_{-\tau_{i j}(0)}^{\omega-\tau_{i j}(\omega)} \sum_{j=1}^{m} \frac{a_{i j}\left(\mu_{i j}(s)\right)}{1-\tau_{i j}^{\prime}\left(\mu_{i j}(s)\right)} \exp \left\{u_{1}(s)\right\} d s \\
= & \int_{0}^{\omega} \sum_{j=1}^{m} \frac{a_{i j}\left(\mu_{i j}(s)\right)}{1-\tau_{i j}^{\prime}\left(\mu_{i j}(s)\right)} \exp \left\{u_{1}(s)\right\} d s,(i=1,2 ; j=1,2, \ldots, m),(10) \\
\int_{0}^{\omega} & \sum_{j=1}^{m} b_{i j}(t) \exp \left\{u_{2}\left(t-\rho_{i j}(t)\right)\right\} d t \\
= & \int_{-\rho_{i j}(0)}^{\omega-\rho_{i j}(\omega)} \sum_{j=1}^{m} \frac{b_{i j}\left(\varepsilon_{i j}(s)\right)}{1-\rho_{i j}^{\prime}\left(\varepsilon_{i j}(s)\right)} \exp \left\{u_{2}(s)\right\} d s \\
= & \int_{-\rho_{i j}(0)}^{\omega-\rho_{i j}(\omega)} \sum_{j=1}^{m} \frac{b_{i j}\left(\varepsilon_{i j}(s)\right)}{1-\rho_{i j}^{\prime}\left(\varepsilon_{i j}(s)\right)} \exp \left\{u_{2}(s)\right\} d s \\
= & \int_{0}^{\omega} \sum_{j=1}^{m} \frac{b_{i j}\left(\varepsilon_{i j}(s)\right)}{1-\rho_{i j}^{\prime}\left(\varepsilon_{i j}(s)\right)} \exp \left\{u_{2}(s)\right\} d s,(i=1,2 ; j=1,2, \ldots, m) .(11)
\end{aligned}
$$


From (9) (10) (11), we can obtain

$$
\begin{gathered}
\int_{0}^{\omega} \sum_{j=1}^{m} \frac{a_{1 j}\left(\mu_{1 j}(s)\right)}{1-\tau_{1 j}^{\prime}\left(\mu_{1 j}(s)\right)} \exp \left\{u_{1}(s)\right\} d s+\int_{0}^{t} \sum_{j=1}^{m} \frac{b_{1 j}\left(\varepsilon_{1 j}(s)\right)}{1-\rho_{1 j}^{\prime}\left(\varepsilon_{1 j}(s)\right)} \exp \left\{u_{2}(s)\right\} d s=\overline{r_{1}} \omega \\
\int_{0}^{\omega} \sum_{j=1}^{m} \frac{a_{2 j}\left(\mu_{2 j}(s)\right)}{1-\tau_{2 j}^{\prime}\left(\mu_{2 j}(s)\right)} \exp \left\{u_{1}(s)\right\} d s-\int_{0}^{\omega} \sum_{j=1}^{m} \frac{b_{2 j}\left(\varepsilon_{2 j}(s)\right)}{1-\rho_{2 j}^{\prime}\left(\varepsilon_{2 j}(s)\right)} \exp \left\{u_{2}(s)\right\} d s=-\overline{r_{2}} \omega .
\end{gathered}
$$

By the mean value theorem for improper integral, there exist $\xi_{i k j} \in[0, \omega](i=1,2 ; k=1,2 ; j=1,2, \ldots, m)$ such that

$$
\begin{aligned}
& A_{11} \int_{0}^{\omega} \exp \left\{u_{1}(s)\right\} d s+A_{12} \int_{0}^{\omega} \exp \left\{u_{2}(s)\right\} d s=\overline{r_{1}} \omega \\
& A_{21} \int_{0}^{\omega} \exp \left\{u_{1}(s)\right\} d s-A_{22} \int_{0}^{\omega} \exp \left\{u_{2}(s)\right\} d s=-\overline{r_{2}} \omega
\end{aligned}
$$

where

$$
\begin{aligned}
& A_{11}=\sum_{j=1}^{m} \frac{a_{1 j}\left(\xi_{11 j}\right) \mu_{1 j}\left(\xi_{11 j}\right)}{1-\tau_{1 j}^{\prime}\left(\mu_{1 j}\left(\xi_{11 j}\right)\right)}, \\
& A_{12}=\sum_{j=1}^{m} \frac{b_{1 j}\left(\xi_{12 j}\right) \varepsilon_{1 j}\left(\xi_{12 j}\right)}{1-\rho_{1 j}^{\prime}\left(\varepsilon_{1 j}\left(\xi_{12 j}\right)\right)}, \\
& A_{21}=\sum_{j=1}^{m} \frac{a_{2 j}\left(\xi_{21 j}\right) \mu_{2 j}\left(\xi_{21 j}\right)}{1-\tau_{2 j}^{\prime}\left(\mu_{2 j}\left(\xi_{21 j}\right)\right)}, \\
& A_{22}=\sum_{j=1}^{m} \frac{b_{2 j}\left(\xi_{22 j}\right) \varepsilon_{2 j}\left(\xi_{22 j}\right)}{1-\rho_{2 j}^{\prime} \varepsilon_{2 j}\left(\left(\xi_{22 j}\right)\right)} .
\end{aligned}
$$

Then,

So we have

$$
\begin{aligned}
& \int_{0}^{\omega} \exp \left\{u_{1}(s)\right\} d s=\frac{\left(A_{22} \overline{r_{1}}-A_{12} \overline{r_{2}}\right) \omega}{A_{11} A_{22}+A_{21} A_{12}} \\
& \int_{0}^{\omega} \exp \left\{u_{2}(s)\right\} d s=\frac{\left(A_{21} \overline{r_{1}}+A_{11} \overline{r_{2}}\right) \omega}{A_{11} A_{22}+A_{21} A_{12}}
\end{aligned}
$$

$$
\begin{aligned}
& \frac{\left(D_{22}^{L}{\overline{r_{1}}}^{L}-D_{12}^{M}{\overline{r_{2}}}^{M}\right) \omega}{D_{11}^{M} D_{22}^{M}+D_{21}^{M} D_{12}^{M}} \leq \int_{0}^{\omega} \exp \left\{u_{1}(s)\right\} d s \leq \frac{\left(D_{22}^{M}{\overline{r_{1}}}^{M}-D_{12}^{L} \bar{r}^{L}\right) \omega}{D_{11}^{L} D_{22}^{L}+D_{21}^{L} D_{12}^{L}}, \\
& \frac{\left(D_{21}^{L}{\overline{r_{1}}}^{L}+D_{11}^{M}{\overline{r_{2}}}^{M}\right) \omega}{D_{11}^{M} D_{22}^{M}+D_{21}^{M} D_{12}^{M}} \leq \int_{0}^{\omega} \exp \left\{u_{2}(s)\right\} d s \leq \frac{\left(D_{21}^{M} \bar{r}_{1}^{M}+D_{11}^{L} \bar{r}_{2}^{L}\right) \omega}{D_{11}^{L} D_{22}^{L}+D_{21}^{L} D_{12}^{L}},
\end{aligned}
$$

where

$$
\begin{aligned}
& D_{11}=\sum_{j=1}^{m} \frac{a_{1 j}(t) \mu_{1 j}(t)}{1-\tau_{1 j}^{\prime}\left(\mu_{1 j}(t)\right)}, \\
& D_{12}=\sum_{j=1}^{m} \frac{b_{1 j}(t) \varepsilon_{1 j}(t)}{1-\rho_{1 j}^{\prime}\left(\varepsilon_{1 j}(t)\right)}, \\
& D_{21}=\sum_{j=1}^{m} \frac{a_{2 j}(t) \mu_{2 j}(t)}{1-\tau_{2 j}^{\prime}\left(\mu_{2 j}(t)\right)}, \\
& D_{22}=\sum_{j=1}^{m} \frac{b_{2 j}(t) \varepsilon_{2 j}(t)}{1-\rho_{2 j}^{\prime}\left(\varepsilon_{2 j}(t)\right)} .
\end{aligned}
$$

By the condition of theorem 3.1, there exist $t_{i} \in[0, \omega], i=1,2$ such that

$$
\begin{aligned}
& u_{1}\left(t_{1}\right)=\ln \left[\frac{\left(A_{22} \overline{r_{1}}-A_{12} \overline{r_{2}}\right) \omega}{A_{11} A_{22}+A_{21} A_{12}}\right], \\
& u_{2}\left(t_{2}\right)=\ln \left[\frac{\left(A_{21} \overline{r_{1}}+A_{11} \overline{r_{2}}\right) \omega}{A_{11} A_{22}+A_{21} A_{12}}\right] .
\end{aligned}
$$


By the condition $\left(H_{4}\right)$ of theorem 3.1, there exist $B_{1}, B_{2}>0$ such that

$$
\left|u_{1}\left(t_{1}\right)\right| \leq B_{1},\left|u_{2}\left(t_{2}\right)\right| \leq B_{2} .
$$

In view of the following:

$$
\begin{aligned}
& \int_{0}^{\omega}\left|\dot{u}_{1}(t)\right| d t=\lambda \int_{0}^{\omega} \mid\left[r_{1}(t)-\sum_{j=1}^{m} a_{1 j}(t) \exp \left\{u_{1}\left(t-\tau_{1 j}(t)\right)\right\}\right. \\
& \left.-\sum_{j=1}^{m} b_{1 j}(t) \exp \left\{u_{2}\left(t-\rho_{1 j}(t)\right)\right\}\right] \mid d t \\
& \leq 2 \int_{0}^{\omega}\left|r_{1}(t)\right| d t=2 \int_{0}^{\omega} r_{1}(t) d t=2 \overline{r_{1}} \omega:=B_{3}, \\
& \int_{0}^{\omega}\left|\dot{u}_{2}(t)\right| d t=\lambda \int_{0}^{\omega} \mid\left[r_{2}(t)+\sum_{j=1}^{m} a_{2 j}(t) \exp \left\{u_{1}\left(t-\tau_{2 j}(t)\right)\right\}\right. \\
& \left.-\sum_{j=1}^{m} b_{2 j}(t) \exp \left\{u_{2}\left(t-\rho_{2 j}(t)\right)\right\}\right] d t \\
& \leq \overline{r_{2}} \omega+\int_{0}^{\omega}\left|\sum_{j=1}^{m} a_{2 j}(t) \exp \left\{u_{1}\left(t-\tau_{2 j}(t)\right)\right\}\right| d t \\
& +\int_{0}^{\omega}\left|\sum_{j=1}^{m} b_{2 j}(t) \exp \left\{u_{2}\left(t-\rho_{2 j}(t)\right)\right\}\right| d t \\
& \leq \overline{r_{2}} \omega+\sum_{j=1}^{m} \frac{a_{2 j}\left(\mu_{2 j}(s)\right) \omega}{1-\mu_{2 j}^{\prime}\left(\mu_{2 j}(s)\right)} \int_{0}^{\omega} \exp \left\{u_{1}(s)\right\} d s \\
& +\sum_{j=1}^{m} \frac{b_{2 j}\left(\varepsilon_{2 j}(s)\right) \omega}{1-\rho_{2 j}^{\prime}\left(\varepsilon_{2 j}(s)\right)} \int_{0}^{\omega} \exp \left\{u_{2}(s)\right\} d s . \\
& \leq \overline{r_{2}} \omega+\sum_{j=1}^{m} \frac{a_{2 j}\left(\mu_{2 j}(s)\right) \omega}{1-\mu_{2 j}^{\prime}\left(\mu_{2 j}(s)\right)} \frac{\left(A_{22}^{M} \bar{r}_{1}^{M}-A_{12}^{L} \bar{r}_{2}^{L}\right) \omega}{A_{11}^{L} A_{22}^{L}+A_{21}^{L} A_{12}^{L}} \\
& +\sum_{j=1}^{m} \frac{b_{2 j}\left(\varepsilon_{2 j}(s)\right) \omega}{1-\rho_{2 j}^{\prime}\left(\varepsilon_{2 j}(s)\right)} \frac{\left(A_{21}^{M}{\overline{r_{1}}}^{M}+A_{11}^{L}{\overline{r_{2}}}^{L}\right) \omega}{A_{11}^{L} A_{22}^{L}+A_{21}^{L} A_{12}^{L}}:=B_{4},
\end{aligned}
$$

then it follows from (22) (23) (24) that

$$
\begin{aligned}
& \left|u_{1}(t)\right| \leq\left|u_{1}\left(t_{1}\right)\right|+\int_{0}^{\omega}\left|u_{1}(t)\right| d t \leq B_{1}+B_{3}:=B_{5}, \\
& \left|u_{2}(t)\right| \leq\left|u_{2}\left(t_{2}\right)\right|+\int_{0}^{\omega}\left|u_{2}(t)\right| d t \leq B_{2}+B_{4}:=B_{6} .
\end{aligned}
$$

Obviously, $B_{1}, B_{2}, B_{3}, B_{4}$ are independent of $\lambda \in(0,1)$. By the condition $\left(H_{5}\right)$ of Theorem 3.1 , it is easy to show that the algebraic equations

$$
\left\{\begin{array}{l}
\sum_{i=1}^{m} \overline{a_{1 j}} u_{1}+\sum_{i=1}^{m} \overline{b_{1 j}} u_{2}=\overline{r_{1}}, \\
\sum_{i=1}^{m} \overline{a_{2 j}} u_{1}-\sum_{i=1}^{m} \overline{b_{2 j}} u_{2}=-\overline{r_{2}},
\end{array}\right.
$$

has a unique positive solution $\left(\tilde{u_{1}}, \tilde{u_{2}}\right)^{T} \in R^{2}$. Take $M=\max \left\{B_{5}, B_{6}\right\}+B_{0}$, where $B_{0}$ is taken sufficiently large such that the unique positive solution $\left(\tilde{u}_{1}, \tilde{u}_{2}\right)^{T} \in R^{2}$ satisfies $\max _{t \in[0, \omega]}\left|\tilde{u}_{1}\right|+\max _{t \in[0, \omega]}\left|\tilde{u}_{2}\right|<B_{0}$,

Let $\Omega:=\{u=\{u(t)\} \in X:\|u\|<M\}$, then it is easy to see that $\Omega$ is an open, bounded set in $X$ and verifies requirement (a) of Lemma 2.1. When $\left(u_{1}(t), u_{2}(t)\right)^{T} \in \partial \Omega \cap \operatorname{Ker} L=\partial \Omega \cap R^{2}, u=\left\{\left(u_{1}, u_{2}\right)^{T}\right\}$ is a constant vector in $R^{2}$ with $\|u\|=$ 
$\left\|\left(u_{1}(t), u_{2}(t)\right)^{T}\right\|=\max _{t \in[0, \omega]}\left|u_{1}(t)\right|+\max _{t \in[0, \omega]}\left|u_{2}(t)\right|=M$. Then we have

$$
Q N y=\left(\begin{array}{l}
\overline{r_{1}}-\sum_{i=1}^{m} \overline{a_{1 j}} u_{1}-\sum_{i=1}^{m} \overline{b_{1 j}} u_{2} \\
\overline{r_{2}}+\sum_{i=1}^{m} \overline{a_{2 j}} u_{1}-\sum_{i=1}^{m} \overline{b_{2 j}} u_{2}
\end{array}\right) \neq 0 .
$$

Letting $J$ be the identity mapping and by direct calculation, we get

$$
\begin{aligned}
& \operatorname{deg}\left\{\operatorname{JQN}\left(u_{1}, u_{2}\right)^{T} ; \partial \Omega \bigcap \text { kerL; } 0\right\} \\
= & \operatorname{deg}\left\{Q N\left(u_{1}, u_{2}\right)^{T} ; \partial \Omega \bigcap \text { kerL } ; 0\right\} \\
= & \operatorname{sign}\left\{\operatorname{det}\left(\begin{array}{cc}
-\sum_{i=1}^{m} \overline{a_{1 j}} & -\sum_{i=1}^{m} \overline{b_{1 j}} \\
\sum_{i=1}^{m} \overline{a_{2 j}} & -\sum_{i=1}^{m} \overline{b_{2 j}}
\end{array}\right)\right\} \\
= & \operatorname{sign}\left\{\sum_{i=1}^{m} \overline{a_{1 j}} \sum_{i=1}^{m} \overline{b_{2 j}}+\sum_{i=1}^{m} \overline{a_{2 j}} \sum_{i=1}^{m} \overline{b_{1 j}}\right\}=1 \neq 0 .
\end{aligned}
$$

This proves that condition (b) in Lemma 2.1 is satisfied. By now, we have proved that $\Omega$ verifies all requirements of Lemma 2.1, then it follows that $L u=N u$ has at least one solution $\left(u_{1}(t), u_{2}(t)\right)^{T}$ in $\operatorname{Dom} L \cap \bar{\Omega}$, that is to say, (5) has at least one $\omega$ periodic solution in $\operatorname{Dom} L \cap \bar{\Omega}$. Then we know that $\left((x(t), y(t))^{T}=\left(\exp \left\{u_{1}(t)\right\}, \exp \left\{u_{2}(t)\right\}\right)^{T}\right.$ is an $\omega$ periodic solution of system (2) (3) with strictly positive components. This completes the proof.

Remark 3.1. Theorem 3.1 remains valid if some or all terms are replaced by corresponding terms with discrete time delays, distribute delays (finite or infinite), or deviating arguments respectively. At this point, we would like to point out that, when one applies the continuation theorem from the coincidence degree theory to explore the existence of periodic solutions to the system of differential equations or difference equations, time delays of any type or the deviating arguments have no effect on the existence of positive solutions.

\section{References}

Chen, Shihua, Wang, Tiaoxiao \& Zhang, Junhua. (2004). Positive Periodic Solution for Non-autonomous Competition Lotka-Volterra Patch System with Time Delay. Nonlinear Analysis: Real World Applications, No. 5, p.409-419.

Ding, Wei \& Han, Maoan. (2008). Dynamic of Non-autonomous Predator -Prey System with Infinite Delay and Diffusion. Computers \& Mathematics with Applications, No. 56, p.1335-1350.

Ding, Xiaoquan, \& Wang, Fangfang. (2008). Positive Periodic Solution for a Semi-ratio-dependent Predator-prey System with Diffusion and Time Delays. Nonlinear Analysis: Real World Applications, No. 9, 239-249.

Fan, Meng \& Kuang, Yang. (1999). Existence and Global Attractivity of Positive Periodic Solutions of Periodic N-species Lotka-Volterra Competition Systems with Several Deviating Arguments. Mathematical Biosciences, No. 160, p. 47-61.

Li, Yongkun\& Kuang, Yang. (2001). Periodic Solutions of Periodic Delay Lotka-Volterra Equations and Systems. Journal of Mathematical Analysis and Applications, No. 9, p.255-260.

Mostafa, Fazly \& Mahmoud, Hesaaraki. (2008). Periodic Solutions for Predator -prey Systems with BeddingtonDeAngelis Functional Response on Time Scales. Nonlinear Analysis: Real World Applications, No. 9, p. 1244-1235.

Robert E. Gaines \& Jean L. Mawhin. (1997). Coincidence Degree and Nonlinear Differential Equations. Springer-verlag Berlin. Journal of Mathematical Analysis and Applications.

Wang, Linlin\& Li, Wantong. (2004). Periodic Solutions and Permeance for a Delayed Non-autonomous Ratio-dependent Predator-prey model with Holling Type Functional Response. Journal of Computational and Applied Mathematics. No. 162, p. 341-357.

Wang, Wending \& Ma, Zhien. (1991). Harmless Delays for Uniform Persistence. Journal of Mathematical Analysis and Applications, No.158. p. 256-268.

Wang, Wending, Zhang, Peihong, Zhu, Deming \& Bi, Ping. (2007). Multiple Positive Solutions of a Delayed Discrete Predator-Prey System with Type IV Functional Responses. Applied Mathematics Letters, No.20. p. 1031-1038.

Xiaohua Ding, Chun Lu \& Mingzhu Liu. (2008). Periodic Solutions for a Semi-Ratio-Dependent Predator-Prey System with Non-monotonic Functional Response and Time Delay. Nonlinear Analysis: Real World Applications, No. 9, p.762775. 
Xinmin Wu, Jingwen Li, \& Wang, zhicheng. (2008). Existence of Positive Solutions for a Generalized Predator-prey model with Harvesting Term. Computers $\mathcal{F}$ Mathematics with Applications, No. 55, p. 1895-1905.

Yang, Shoujie, Shi, Bao. (2008). Periodic Solution for a Three-Stage-Structured Predator-Prey System with Delay. Journal of Mathematical Analysis and Applications, No. 341, p.287-294.

Yang, Zhihui \& Cao, Jinde. (2007). Periodic Solutions for General Nonlinear State-Dependent Delay Logistic Equations. Nonlinear Anal., No. 66, p.1378-1387.

Zhang, Weipeng, Bi, Ping \& Zhu, Deming. (2008). Periodicity in a Ratio-dependent Predator -prey System with Stagestructured Predator on Time Scales. Nonlinear Analysis: Real World Applications, No. 9, p. 344-353.

Zhang, Zhengqiu, Hou, Zhenting \& Wang, Lin. (2008). Multiplicity of Positive Periodic Solutions to a Generalized Delayed Predator-Prey System with Stocking. Nonlinear Analysis: Real World Applications, No. 68, p.2608-2622.

Zhang, Zhengqiu \& Zheng, Xianwu. (2003). On a Periodic Stage-Structure Model. Applied Mathematics Letters, No.16(7). p.1053-1061. 